University of South Carolina

Scholar Commons

$9-9-2010$

\title{
Testing Photons' Bose-Einstein Statistics With Compton Scattering
}

Brett David Altschul

altschul@mailbox.sc.edu

Follow this and additional works at: https://scholarcommons.sc.edu/phys_facpub

Part of the Physics Commons

Publication Info

Published in Physical Review D, Volume 82, Issue 10, 2010.

(C) 2010 The American Physical Society

This Article is brought to you by the Physics and Astronomy, Department of at Scholar Commons. It has been accepted for inclusion in Faculty Publications by an authorized administrator of Scholar Commons. For more information, please contact digres@mailbox.sc.edu. 


\title{
Testing Photons' Bose-Einstein Statistics With Compton Scattering
}

\author{
Brett Altschul] \\ Department of Physics and Astronomy \\ University of South Carolina \\ Columbia, SC 29208
}

\begin{abstract}
It is an empirical question whether photons always obey Bose-Einstein statistics, but devising and interpreting experimental tests of photon statistics can be a challenge. The nonrelativistic cross section for Compton scattering illustrates how a small admixture $\nu$ of wrong-sign statistics leads to a loss of gauge invariance; there is a large anomalous amplitude for scattering timelike photons. Nevertheless, one can interpret the observed transparency of the solar wind plasma at low frequencies as a bound $\nu<10^{-25}$ if Lorentz symmetry is required. If there is instead a universal preferred frame, the bound is $\nu<$ $10^{-14}$, still strong compared with previous results.
\end{abstract}

\footnotetext{
${ }^{1}$ baltschu@physics.sc.edu
} 
The connection between spin and statistics is one of the most profound results in quantum physics. In a relativistic quantum theory, imposing a small number of reasonable conditions (including Lorentz invariance and stability of the vacuum state) necessitates that particles with integer spin have wave functions symmetric under exchange and those with half integer spin have antisymmetric wave functions. This is a theorem in axiomatic field theory, but whether it is really true of physical particles is a separate question. If any deviation from the usual spin-statistics relationship were uncovered in the laboratory, this would be a discovery of profound importance; it would be a sure sign of genuinely new physics, and it would necessitate a violation of some other basic axiom that has been believed to hold.

Recently, a remarkable experiment using a two-photon atomic transition has been interpreted as a new test of photon statistics [1]. The transition rate is affected by the symmetry state of the two photons involved. However, the strategy used in this laboratory test can actually be applied to a much broader class of experiments, and it is important that a violation of Bose-Einstein (BE) statistics would not be a purely quantum mechanical effect. Classical electrodynamics already contains many of the correlations that characterize $\mathrm{BE}$ behavior. It is therefore possible to test the statistics of photons by looking only at classical (i.e. tree level) processes.

Studies of spin-statistics violations have historically focused more on small deviations from Fermi-Dirac (FD) statistics. There are two good reasons for this. First, it is quite easy to conceive of an experiment to test the Pauli Exclusion Principle: one simply tests whether it is genuinely impossible for two electrons to occupy the same quantum state, something that can be determined from atomic spectroscopy [2]. Second, the classical limit of a fermionic field means having a single quantum present, while the classical limit of a bosonic field involves a large number of quanta. But while it is clearly meaningful to have a bosonic field with only one quanta present, one cannot have a fermionic field with many. This means that a small change in the statistics of a spin- $\frac{1}{2}$ field need not have large, obvious consequences in the classical limit; it is easier to envision a form of new physics that occasionally allows more than one particle in a given state than a new physical principle that occasionally reduces the allowed number from infinity to something finite. This makes testing photons statistics an interesting challenge.

The experiment in [1] used two laser beams, aligned nearly parallel. The two beams' frequencies were swept up and down, keeping their sum constant and in resonance with a $\Delta J=1$ atomic transition. Most of the time, the photons could combine (with one from each beam) to excite this transition. However, the requirement that two photons be in a symmetric state dictates that two photons with equal momenta cannot be in a state with total angular momentum $J=1$. Therefore, if the two laser frequencies were equal, the atomic transition rate would vanish. As the frequencies approached equality, $\mathrm{BE}$ correlations ensured the transition rate went smoothly to zero. The object of study was these correlations; the experiment did not look directly at the question of whether it is possible for multiple photons to be present in the same quantum state. 
If the BE correlations were not present exactly as expected, one would expect there to be a small but nonzero transition rate even if photons from the two laser beams had precisely equal momenta. Such a modification to the photons' statistics was modeled as follows. In the transition rate, there are two diagrams, in which the photons are absorbed in opposite orders. Instead of simply calculating the sum of the two diagrams, there could be a small admixture of the difference of the diagrams. This difference would normally be taken if photons were fermions, so this model means having a small admixture of wrong-sign statistics. In addition to the usual transition rate $w_{B E}$ (calculated with $\mathrm{BE}$ statistics), there is a small fraction $\nu$ of the FD transition rate $w_{F D}$, making the total rate $w=w_{B E}+\nu w_{F D}$.

Note that the test described in [1] does not involve electromagnetic excitations that can be treated as non-bosonic over their entire time of existence. Instead, the electromagnetic field is prepared in a coherent and intrinsically bosonic laser state. In models in which there exists a small admixture of FD photons among the usual BE ones, the use of lasers may be problematic. The techniques used to enhance the intensity of a laser beam may increase the number of bosonic photons present, but the number of fermionic photons will remain zero or one. Thus, increasing the laser power may enhance only the absorption rate for conventional photons, and the FD signal may be swamped by BE noise.

For this reason, the two-photon absorption measurement is most sensitive to the possibility of photons that behave, for the vast majorities of the lifetimes, as bosons; however, they briefly behave according to FD statistics in the time period immediately surrounding the atomic transition. This restricts the applicability of these bounds to only certain types of spin-statistics violations. This restriction will apply to any experimental technique that uses large electromagnetic field amplitudes.

Compton scattering, viewed as a scattering process with one fermion and one photon, need not suffer from the same limitation. The photon could be entirely fermionic, over the entire course of its existence. This makes Compton scattering (and other scattering processes that do not involve coherent assemblages of photons) sensitive to more possible forms of spin-statistics violation than tests with lasers or macroscopic fields. However, the modified Compton scattering cross section can also be applied to situations with coherent fields, such as the scattering of radio waves in diffuse plasmas. In that case, a study using Compton scattering would be sensitive to the same kinds of statistical correlations as the two-photon absorption experiment.

No complete theory in which photons do not obey BE statistics is known. We shall therefore follow the interpretational methodology of [1]; we consider a single process, which receives contributions from two distinct photon interaction diagrams. The statistical properties of the electromagnetic excitations involved determine the relative sign between the two diagrams. Depending on the statistics, the amplitudes may interfere constructively or destructively. We shall show, by example, that inverting the relative sign between the two diagrams that can contribute to an $\mathcal{O}\left(e^{2}\right)$ interaction amplitude destroys a number of key properties of quantum electrodynamics. Gauge invariance is lost, 
as may be Lorentz invariance. Nevertheless, we can estimate how strongly the violation of BE statistics for photons is constrained by experiment.

The matrix element squared for Compton scattering (summed and averaged over fermion polarizations, and using natural units in which $\hbar=c=\varepsilon_{0}=1$ ) is

$$
\begin{aligned}
\frac{1}{2} \sum_{f \text { spins }}|\mathcal{M}|^{2}= & \frac{e^{4}}{2}\left(\epsilon_{\mu}^{\prime *} \epsilon_{\rho}^{\prime}\right)\left(\epsilon_{\nu} \epsilon_{\sigma}^{*}\right) \operatorname{tr}\left[\left(\not p^{\prime}+m\right)\left(\frac{\gamma^{\mu} \not k \gamma^{\nu}+2 \gamma^{\mu} p^{\nu}}{2 p \cdot k} \pm \frac{\gamma^{\nu} \not k^{\prime} \gamma^{\mu}-2 \gamma^{\nu} p^{\mu}}{2 p \cdot k^{\prime}}\right)\right. \\
& \left.\times(\not p+m)\left(\frac{\gamma^{\sigma} \not k \gamma^{\rho}+2 \gamma^{\rho} p^{\sigma}}{2 p \cdot k} \pm \frac{\gamma^{\rho} \not k^{\prime} \gamma^{\sigma}-2 \gamma^{\sigma} p^{\rho}}{2 p \cdot k^{\prime}}\right)\right] .
\end{aligned}
$$

$p$ and $p^{\prime}$ are the initial and final fermion momenta; $k$ and $k^{\prime}$ are the corresponding photon momenta; $\epsilon(k)$ and $\epsilon^{\prime}\left(k^{\prime}\right)$ are the incoming and outgoing polarization vectors. The + signs between the terms are the usual ones; the - signs correspond to the possibility of inverted statistics. There are two Feynman diagrams that contribute to this process; they differ in the order of the photon emission and absorption vertices along the fermion line. (In nonrelativistic scattering with the wrong photon statistics, this is the same as the time order of the two photon interaction events.) Normally the two diagrams are summed, in accordance with BE statistics.

We shall concentrate on the low-energy regime, in which the fermion is initially at rest and $\omega \ll m$. Energy-momentum conservation then dictates that $\omega^{\prime} \approx \omega$ and $p^{\prime} \approx$ $p=(m, \overrightarrow{0})$. The largest contribution to the scattering cross section then comes from the electron propagator terms with $p$ in the numerator. Since $p \cdot k^{\prime} \approx p \cdot k=m \omega$, this becomes

$$
\begin{aligned}
\frac{1}{2} \sum_{f \text { spins }}|\mathcal{M}|^{2}= & \frac{e^{4}}{2}\left(\epsilon_{\mu}^{*} \epsilon_{\rho}^{\prime}\right)\left(\epsilon_{\nu} \epsilon_{\sigma}^{*}\right) \operatorname{tr}\left\{\left(m \gamma^{0}+m\right)\left[\frac{2 \gamma^{\mu}\left(m g^{0 \nu}\right)}{2 m \omega} \pm \frac{-2 \gamma^{\nu}\left(m g^{0 \mu}\right)}{2 m \omega}\right]\right. \\
& \left.\times\left(m \gamma^{0}+m\right)\left[\frac{2 \gamma^{\rho}\left(m g^{0 \sigma}\right)}{2 m \omega} \pm \frac{-2 \gamma^{\sigma}\left(m g^{0 \rho}\right)}{2 m \omega}\right]\right\} \\
= & \left(\epsilon_{\mu}^{\prime *} \epsilon_{\rho}^{\prime}\right)\left(\epsilon_{\nu} \epsilon_{\sigma}^{*}\right) \frac{e^{4} m^{2}}{2 \omega^{2}} \operatorname{tr}\left[\left(\gamma^{0}+1\right) \gamma^{\mu}\left(\gamma^{0}+1\right) \gamma^{\rho} g^{0 \nu} g^{0 \sigma}\right. \\
& \mp(\mu \leftrightarrow \nu) \mp(\rho \leftrightarrow \sigma)+(\mu \leftrightarrow \nu, \rho \leftrightarrow \sigma)] .
\end{aligned}
$$

Since $\operatorname{tr}\left[\left(\gamma^{0}+1\right) \gamma^{\mu}\left(\gamma^{0}+1\right) \gamma^{\rho}\right]=8 g^{0 \mu} g^{0 \rho}$, the matrix element squared is

$$
\frac{1}{2} \sum_{f \text { spins }}|\mathcal{M}|^{2}=\left(\epsilon_{\mu}^{*} \epsilon_{\rho}^{\prime}\right)\left(\epsilon_{\nu} \epsilon_{\sigma}^{*}\right) \frac{16 e^{4} m^{2}}{\omega^{2}} g^{0 \mu} g^{0 \nu} g^{0 \rho} g^{0 \sigma}
$$

for wrong-sign statistics, while it vanishes (at this order in $\frac{m}{\omega}$ ) for BE statistics. The total cross section - including both the usual term and a small admixture $\nu$ of wrongsign statistics - is $\sigma=\sigma_{T}\left(1+6 \frac{m^{2}}{\omega^{2}} \Sigma \nu\right)$, where $\sigma_{T}=\frac{8 \pi \alpha^{2}}{3 m^{2}}$ is the Thomson cross section, and $\Sigma=\left|\epsilon_{0}^{\prime}\right|^{2}\left|\epsilon_{0}\right|^{2}$ represents the photon polarization expression, which must be properly 
interpreted. The result remains unchanged if the scatterer is a composite particle with form factors $F_{1}$ and $F_{2}$.

The matrix element for the wrong statistics case has many unusual features. The scattering is isotropic, in contrast to the usual case, and the cross section is large$\mathcal{O}\left(\frac{m^{2}}{\omega^{2}}\right)$. The contributions that make it large come from virtual intermediate states that are nearly on shell; they have energy defects of approximately $\pm \omega$ and are very close to being real states. Normally, there is destructive interference between the two diagrams, wiping out those terms in the matrix element that diverge as $\omega \rightarrow 0$. This interference is ensured by the BE statistics of the photons; the incident and scattered photons must be in a symmetric state. (Note that, depending on the temporal order in which absorption and emission occur, these two photons may or may not be physically present at the same instant in time; however, the statistical relationship between the two is required whether or not they actually exist simultaneously.) Without the conventional symmetry requirement, the cancellation is not complete, and large new terms appear in the cross section. These terms modify the Thomson result, which is ordinarily entirely classical in character; however, since the large modifications are tied to the presence of nearly on shell virtual states, there is probably no way to formulate the wrong-statistics photon scattering problem using only classical techniques.

Moreover, in the wrong-statistics version, gauge invariance is obviously violated; the amplitude is not transverse to the photon momenta: $k_{\nu} \mathcal{M}^{\mu \nu} \neq 0$. The cancellation between the two diagrams is crucial to maintaining this Ward identity in the conventional case. The modified behavior represents a large deviation from standard physics, and the classical interaction Lagrangian for interactions between the charged particle and the magnetic field is no longer the usual $-e v^{\mu} A_{\mu}$. Gauge invariance is lost if FD statistics are used in the two-photon absorption experiment as well. The loss of gauge symmetry could have even more drastic consequences in other processes; if the wrong-statistics amplitude were included in calculations of the vacuum polarization, that diagram would generate a divergent photon mass.

The $|\mathcal{M}|^{2}$ appearing in (4) corresponds to scattering exclusively to and from timelike photon states; those are ghost states with negative norm. Normally, the contributions from timelike states are precisely canceled by contributions from longitudinal polarization states; this is a consequence of gauge invariance, as expressed by the Ward identity. A sum over polarization vectors $\sum \epsilon_{\nu} \epsilon_{\sigma}^{*}$ may be replaced by $-g_{\nu \sigma}$, which produces a manifestly Lorentz invariant expression.

However, the failure of the Ward identity when the wrong statistics are used makes interpreting (44) a nontrivial matter. If gauge symmetry is broken, there must be an ancillary condition on the electromagnetic field (e.g. the Lorenz condition $\partial^{\mu} A_{\mu}=0$ in massive electrodynamics), but it is not clear what the condition should be in the theory of wrong-statistics photons. The key question is what external photon polarization vectors $\epsilon$ and $\epsilon^{\prime}$ are appropriate for determining the physical cross section. There are two particularly obvious ways of choosing these vectors, which differ in their physical 
consequences. The two possibilities can essentially be characterized by how many external spin states are to be summed over, but it is not clear which (if any) of the interpretations can be assigned completely consistent interpretations.

The first possible interpretation is simply to retain the prescription $\sum \epsilon_{\nu} \epsilon_{\sigma}^{*} \rightarrow-g_{\nu \sigma}$ in the spin-summed cross section. This has the advantage of preserving Lorentz invariance, by summing over polarization vectors in all four possible spacetime directions. This amounts to setting $\Sigma=\frac{1}{2}$ in the spin-averaged cross-section.

The second possibility is to break Lorentz symmetry, which makes it possible to choose two purely spacelike vectors as the only physical external photon polarizations. Breaking Lorentz symmetry might seem like a drastic and unnecessary additional modification of the physics, but in many ways the theory with broken Lorentz symmetry seems to be better behaved than the theory utilizing the Lorentz-invariant polarization prescription described above. The Lorentz-symmetric option invokes additional polarization states, which generally interact with charged matter as strongly (or more strongly) than the usual transverse polarizations. Yet there are only two real electromagnetic field states that come into black body equilibrium on observable time scales. On the other hand, in the absence of Lorentz invariance, these extra states can be excluded from the theory. Since it is only the timelike photon states that appear in the scattering cross section (4), having only purely spacelike polarizations would almost seem to alleviate the problem with (4) entirely. Yet using just two purely spacelike external polarization vectors does not actually address all the problems. There remain three significant challenges for the Lorentz-violating version of this theory.

The first challenge comes from the fact that (4) is only an approximation. There are other novel terms in the wrong-statistics cross section that do affect the scattering of purely spacelike photons; these terms are smaller, lacking the large enhancement factor $\frac{m^{2}}{\omega^{2}}$, but they are still comparable in size to the usual Thomson cross section and thus are potentially observable, even for small values of $\nu$. The second issue derives from the fact this it is, of course, only possible to choose $\epsilon$ and $\epsilon^{\prime}$ as purely spacelike in one particular frame, which would be a preferred frame for the universe. The only likely candidate for such a frame is the rest frame of the cosmic microwave background (CMB), and the solar system is moving relative to the CMB at a speed $v_{C M B} \approx 2 \times 10^{-3}$. If the time components of $\epsilon$ and $\epsilon^{\prime}$ vanish in the CMB frame, the time components will be $\sim v_{C M B}$ in a terrestrial laboratory frame. So the cross section for scattering off a fermion that is stationary in the lab frame will receive a $\Sigma \sim v_{C M B}^{4}$ contribution from (44). This is a suppression of roughly $10^{-11}$, but it does not rule out observable consequences.

The third challenge for the Lorentz-violating scenario is that it must confront the bounds that have already been placed on spin-independent Lorentz symmetry violations in electron-photon interactions. The best tests of boost invariance in such processes use astrophysical synchrotron and inverse Compton data to place bounds at the $10^{-14}-10^{-19}$ levels [3]. However, the reliability of such bounds is questionable if the electron-photon interaction differs from the usual one; the usual inferences about what processes are 
responsible for the observed spectra of given sources may not be valid. The best laboratory bounds are based on observations of similar processes at the Large Electron-Positron Collider (LEP). Those bounds should be considered more secure, since the processes involved are known with relative certainty. The best LEP bounds on the same quantities are at the $10^{-12}-10^{-15}$ levels [4, 5]. It is not precisely clear how these bounds apply to the Lorentz-violating theory of wrong-statistics photons, but some effect would be expected.

Despite these challenges, it is still possible to estimate how much FD statistics can be present in Compton scattering processes, by looking at the scattering of low-energy photons. For this purpose, it is advantageous to look at the long-distance propagation of radio waves through plasmas. (Diffuse plasmas are preferable, since collective behavior takes over at frequencies below $\omega_{p}=\sqrt{n_{e} e^{2} / m}$, where $n_{e}$ is the electron density.) In order to have a substantial amount of anomalous, frequency-dependent scattering, the optical depth due to the wrong-statistics process, $6 \frac{m^{2}}{\omega^{2}}\left(n L \sigma_{T}\right) \Sigma \nu$ (where $n$ is the density of scatterers, so $n L \sigma_{T}$ is the depth due to Thomson scattering) must be $\sim 1$ or larger.

The Wind satellite observed radio emissions down to frequencies $\frac{\omega}{2 \pi} \approx 70 \mathrm{kHz}$ coming from Earth (over a distance $L>1.3 \times 10^{11} \mathrm{~cm}$ ) with no unusual scattering by the solar wind evident [6]. Since the anomalous scattering is stronger for heavier particles, the relevant density $n$ is that of protons in the solar wind, which was $n_{p} \approx 10-20 \mathrm{~cm}^{-3}$ along the radiation's path. The transparency implies the bound

$$
\Sigma \nu<\frac{\omega^{2}}{6 m_{p}^{2} n_{p} L \sigma_{T}}<1.8 \times 10^{-26} .
$$

For the version of the theory with broken Lorentz symmetry, in which the CMB frame is the preferred frame, this implies a conservative bound on $\nu$ of

$$
\nu<10^{-14} \text {. }
$$

The Lorentz-invariant theory, with strongly coupled longitudinal and timelike photons, is harder to interpret, but the numerical bounds on $\nu$ are stronger,

$$
\nu<10^{-25} \text {. }
$$

Each of these bounds is much stronger than the $\nu<4 \times 10^{-11}$ result from [1].

Of course, the two-photon absorption and Compton scattering data do not measure precisely the same thing. In each case, the rate for a process is calculated by conventional means, except that a possible change in the photons statistics is inserted at one point (with the remainder of the calculation tacitly assuming BE behavior). In the absence of any underlying theory to motivate the change in statistics, it is tricky to relate the results of significantly different experiments (although if there is violation of BE statistics in one case, it would be natural to expect a similar violation in other experiments). So the two-photon absorption and Compton scattering bounds should be seen as complementary, rather than directly competitive. 
Moreover, simple experiments such as these could also be interpreted of tests of other basic principles; and again, the two experimental scenarios would not generally be sensitive to exactly the same things. For example, the two-photon transition experiment could be interpreted as a test not of photon statistics but of whether photons states always have spin angular momentum $J=1$. In Lorentz-violating or nonlinear theories of electrodynamics, this fact is not assured. Unusual behavior in Compton scattering could also be indicative of a different form of Lorentz violation; even though the Thomson cross section can be derived from a very limited set of assumptions (see e.g. [7]), $\mathcal{O}\left(\omega^{-2}\right)$ modifications are possible if boost symmetry is broken [8].

\section{References}

[1] D. English, V. V. Yashchuk, D. Budker, Phys. Rev. Lett. 104, 253604 (2010).

[2] S. Bartalucci, et al. (VIP collaboration), Phys. Lett. B 641, 18 (2006).

[3] B. Altschul, Phys. Rev. D 74, 083003 (2006).

[4] M. A. Hohensee, R. Lehnert, D. F. Phillips, R. L. Walsworth, Phys. Rev. Lett. 102, 170402 (2009).

[5] B. Altschul, Phys. Rev. D 82, 016002 (2010).

[6] M. L. Kaiser, M. D. Desch, W. M. Farrell, J.-L. Steinberg, M. J. Reiner, Geophys. Res. Lett. 23, 1283 (1996).

[7] W. Thirring, Phil. Mag. 41, 1193 (1950).

[8] B. Altschul, Phys. Rev. D 70, 056005 (2004). 